\title{
Leukemia inhibitory factor receptor negatively regulates the metastasis of pancreatic cancer cells in vitro and in vivo
}

\author{
DING MA*, XIAOQIAN JING*, BAIYONG SHEN*', XINYU LIU, XI CHENG, BINGRUI WANG, \\ ZHIPING FU, CHENGHONG PENG and WEIHUA QIU \\ Department of Surgery, Ruijin Hospital, Shanghai Jiao Tong University School of Medicine, \\ Shanghai 200025, P.R. China
}

Received January 26, 2016; Accepted March 22, 2016

DOI: $10.3892 /$ or.2016.4865

\begin{abstract}
Pancreatic cancer (PC) is one of the leading causes of cancer-related deaths worldwide. Frequent metastasis and recurrence are the main reasons for the poor prognosis of PC patients. Thus, the discovery of new biomarkers and wider insights into the mechanisms involved in pancreatic tumorigenesis and metastasis is crucial. In the present study, we report that leukemia inhibitory factor receptor (LIFR) suppresses tumorigenesis and metastasis of PC cells both in vitro and in vivo. LIFR expression was significantly lower in PC tissues and was associated with local invasion $(\mathrm{P}=0.047)$, lymph node metastasis $(\mathrm{P}=0.014)$ and tumor-node-metastasis (TNM) stage $(\mathrm{P}=0.002)$. Overexpression of LIFR significantly suppressed $\mathrm{PC}$ cell colony formation $(\mathrm{P}=0.005)$, migration $(\mathrm{P}=0.003)$, invasion $(\mathrm{P}=0.010)$ and wound healing ability $(\mathrm{P}=0.013)$ in vitro, while opposing results were observed after LIFR was silenced. Furthermore, animal xenograft and metastasis models confirm that the in vivo results were consistent with the outcomes in vitro. Meanwhile, LIFR inhibited the expression of $\beta$-catenin, vimentin and slug and induced the expression of E-cadherin, suggesting that the epithelial-mesenchymal transition regulation pathway may underlie the mechanism. These results indicate that LIFR negatively regulates the metastasis of PC cells.
\end{abstract}

\section{Introduction}

Pancreatic cancer (PC) is the seventh leading cause of cancer-related death among men and women worldwide (1).

Correspondence to: Professor Chenghong Peng or Professor Weihua Qiu, Department of Surgery, Ruijin Hospital, Shanghai Jiao Tong University School of Medicine, Shanghai Institute of Digestive Surgery, 197 Ruijin Er Road, Shanghai 200025, P.R. China E-mail: drqwh2003@yahoo.com

E-mail: drqwh2003@126.com

${ }^{*}$ Contributed equally

Key words: pancreatic cancer, leukemia inhibitory factor receptor, tumorigenesis, metastasis, epithelial-mesenchymal transition
Despite improvements in surgical techniques and adjuvant medical therapy, the prognosis of PC has not been significantly improved in over four decades (2). It is one of the most devastating malignant diseases with a median survival of 3-6 months and a 5-year survival rate of less than 5\% $(3,4)$. After careful assessment, only $15 \%$ of patients are considered to be candidates for surgical resection and undergo resection with curative intent (5). In addition to massive primary tumors, $\sim 30 \%$ of patients die from locally destructive PC, and the other $70 \%$ may have widespread metastatic disease at the time of death (6). Therefore, the discovery of new biomarkers and wider insights into the mechanisms involved in pancreatic tumorigenesis and metastasis are crucial.

Leukemia inhibitory factor receptor (LIFR) is an integral component of the glycoprotein 130-LIFR complex and participates in signal transduction through the interleukin-6 (IL-6) cytokine family, which includes IL-6, IL-11, cardiotrophin-1, ciliary neurotrophic factor, oncostatin $\mathrm{M}$ and cardiotrophinlike cytokine (7). The biological roles of the IL-6 cytokine family are widely different, ranging from glucose uptake, maintenance of stem cell pluripotency, to modulation of cell proliferation. According to Alisoltani et al and the Oncomine data-mining analysis, downregulation of LIFR expression has been found in several types of cancers, including breast, gastric, colorectal, liver and PC (8). LIFR has been observed in several human malignancies, including medulloblastoma, nasopharyngeal carcinoma, lung, breast and liver cancer (9-13). Chen et al identified LIFR as a metastasis suppressor which exerted its function through the Hippo-YAP pathway (11). Luo et al demonstrated that LIFR negatively regulated the metastasis of hepatocellular carcinoma by regulating the phosphoinositide 3-kinase/AKT pathway (14). However, the precise role of LIFR in PC remains largely unexplored. The purpose of the present study was to explore the possibility of LIFR as a potential molecular marker and therapeutic target for PC.

\section{Materials and methods}

Patient samples, cell lines and tissue microarray. From 2012 to 2014,26 PC patients (15 males and 11 females), ranging from 34 to 72 years of age (mean age, 53.6 years), who underwent radical resections were recruited in the current investigation 
with informed consent. Research consent was approved by the Ethics Committee of Ruijin Hospital, Shanghai Jiao Tong University School of Medicine. All patients were aware of the potential risks and complications of the proposed treatment scheme. The corresponding non-tumor tissues were collected at least $3 \mathrm{~cm}$ away from the margin of the tumor. All the specimens including tumor and paired non-tumor tissues were cut into small pieces and placed in liquid nitrogen immediately. All of the samples were submitted for routine pathologic evaluation and diagnostic confirmation. The human PC cell lines Capan-1, CFPAC-1, SW-1990, BxPC-3 and PANC-1 were purchased from the American Type Culture Collection (ATCC; Manassas, VA, USA) and PATU-8988 was purchased from Nanjing KeyGen Biotech Co., Ltd. (Nanjing, China). All tumor cell lines were cultured in Dulbecco's modified Eagle's medium (DMEM) or RPMI-1640 medium supplemented with $10 \%$ fetal bovine serum (FBS) at $37^{\circ} \mathrm{C}$ in $5 \% \mathrm{CO}_{2}$. Total RNA and genomic DNA were isolated according to previously reported methods (15). Additionally, PC tissue array of 29 patients was purchased from Shanghai Outdo Biotech Co., Ltd (Shanghai, China).

Vector construction and transfection. The full-length cDNA of LIFR was obtained using a reverse transcription kit (Takara) and total RNA was extracted by RT-PCR from human PC tissues using TRIzol reagent kit (Invitrogen, Carlsbad, CA, USA). The primers for the coding sequence (CDS) of double-strand DNA fragments of LIFR were: 5'-GGATCCAT GATGGATATTTACGTATGT-3' (forward) and 5'-ACGCGT TTAATCGTTTGGTTTGTTCTG-3' (reverse), and were subcloned into the pWPI-GFP vector to generate pWPIGFP/LIFR. Transfection of the constructed plasmid and empty vector into PATU-8988 cells was performed using Lipofectamine 2000 (Invitrogen) according to the manufacturer's procedure. Cells that had been transfected with the constructed plasmid were then selected by antibiotic resistance in cell culture medium containing $1,500 \mu \mathrm{g} / \mathrm{ml} \mathrm{G} 418$ to obtain cell strains with stable expression of LIFR. After 6 weeks of culture in the presence of G418, the remaining cells were isolated. Positive stable clones with pWPI-GFP/LIFR were maintained with pWPI-GFP empty vector transfection (PATU$8988 /$ vector) as control.

RNA interference. Based on human LIFR gene data, synthesized DNA nucleotide fragments, encoding shRNA for knockdown of LIFR, were inserted into $\mathrm{pL} / \mathrm{shRNA} / \mathrm{F}$ lentiviral vector to obtain $\mathrm{pL} / \mathrm{shRNA} / \mathrm{shR}$-LIFR. The constructions were further confirmed by DNA sequencing. The packaging of the lentivirus and establishment of stable knockdown cell clones were performed as mentioned above. The sequences of these synthesized oligonucleotides for RNAi LIFR were as follows: 5'-GATCCCCGCTGATTTCTCAACCTCTACATTCAA GAGATGTAGAGGTTGAGAAATCAGCTTTTTGGAA-3' (forward) and 5'-AGCTTTTCCAAAAAGCTGATTTCT CAACCTCTACATCTCTTGAATGTAGAGGTTGAGAA ATCAGCGGG-3' (reverse). Then, the pL/shRNA/shR-LIFR lentiviral vector was transfected into Capan-1 cells. Stable clones (Capan-1/sh) were established by selection with $5 \mu \mathrm{g} / \mathrm{ml}$ blasticidin. The irrelevant nucleotides in sh-NC did not target any annotated human genes and served as a negative control.
Immunohistochemistry. Paraffin-embedded tissue samples from PC specimens underwent a heat pre-treatment of $60^{\circ} \mathrm{C}$ for $1 \mathrm{~h}$, then dewaxed in xylene, rehydrated in a series of ethanol and treated with $0.01 \mathrm{~mol} / 1$ citrate buffer $(\mathrm{pH}$ 6.0) for antigen retrieval. After inhibition of endogenous peroxidase activity for $30 \mathrm{~min}$ with methanol containing $0.3 \% \mathrm{H}_{2} \mathrm{O}_{2}$, the sections were stained with rabbit anti-LIFR antibody (C-19) (1:300; Santa Cruz Biotechnology, Santa Cruz, CA, USA) or the anti-Ki67 antibody (ab15580) (1:100; Abcam, Cambridge, MA, USA) at $4{ }^{\circ} \mathrm{C}$ overnight. For tissue arrays, after being dewaxed, hydrated and blocked of non-specific binding sites, the microarray was incubated with rabbit anti-LIFR antibody (C-19) (1:300; Santa Cruz Biotechnology) at $4^{\circ} \mathrm{C}$ overnight. The following experimental procedure was performed according to the manufacturer's instructions for the LSAB+ kit (Dako, Carpinteria, CA, USA). Three pathologists who were blinded to any patient data independently examined the cellular location of LIFR and compared the staining between the tumor and normal tissues. Immunohistochemistry staining score $=$ positive cell score + staining intensity score . The percentage of positive cells was classified according to five grades (percentage scores): $<10 \%$ (grade 0 ), $10-25 \%$ (grade 1$),>25-50 \%$ (grade 2 ), $>50-75 \%$ (grade 3 ) and $>75 \%$ (grade 4). Immunohistochemical staining intensity was graded as follows: 0 (no staining), 1 (bright yellow), 2 (orange) and 3 (brown). The total scores of $\leq 3,>3-5$, and $\geq 6$ were defined as negative, weak and strong positive, respectively.

Western blotting. Tumor and cell samples were collected and lysed using RIPA buffer (Solarbio, Beijing, China) in the presence of protease inhibitor cocktail and protein concentration was measured by BCA protein assay kit (both from Pierce, Rockford, IL, USA). An equal amount of total cellular protein was electrophoresed by $10 \%$ SDS-PAGE, then transferred to polyvinylidene difluoride (PVDF) membranes. The membranes were blocked with 5\% skim milk for $2 \mathrm{~h}$, and then incubated with primary antibodies overnight at $4^{\circ} \mathrm{C}$. Primary antibodies were as follows: LIFR (C-19) (1:1,000; Santa Cruz Biotechnology), vimentin (D21H3) $(1: 1,000)$, N-cadherin (D4R1H) (1:1,000), $\beta$-catenin (D10A8) $(1: 1,000)$, slug (C19G7) $(1: 1,000)$ (all from Cell Signaling Technology, Danvers, MA, USA) and GAPDH (Abcam). After the membranes were incubated with the secondary antibody for $2 \mathrm{~h}$ at room temperature, the proteins were visualized using an enhanced chemiluminescence detection system (Amersham Biosciences, Piscataway, NJ, USA) according to the manufacturer's protocol.

Colony formation assay. For the colony formation assay, 1,000 cells were layered onto 6 -well plates and cultured at $37^{\circ} \mathrm{C}$ for $\sim 14$ days in order to let the colonies develop. After visible colonies of cells were observed, the cells were washed twice with phosphate-buffered saline (PBS), and fixed with $4 \%$ paraformaldehyde and stained with crystal violet for $30 \mathrm{~min}$. Colonies containing 50 cells or more were counted.

Transwell migration and invasion assays. For the cell migration assay, a total number of $1 \times 10^{5}$ cells were re-suspended in serum-free culture solution and added to the Transwell upper chambers ( $8 \mu \mathrm{m}$; 24-well format; Corning, Lowell, MA, USA). To measure the invasion, the upper chamber's membrane was 
Table I. Comparison of LIFR expression between PC and normal pancreatic tissue $(n=55)$.

\begin{tabular}{|c|c|c|c|c|c|c|c|}
\hline \multirow[b]{2}{*}{ LIFR expression } & \multicolumn{2}{|c|}{ Clinical cases $(n=26)$} & \multicolumn{2}{|c|}{ Tissue microarrays $(n=29)$} & \multicolumn{2}{|c|}{ All $(n=55)$} & \multirow[b]{2}{*}{ P-value } \\
\hline & $\mathrm{PC}$ & Normal & $\mathrm{PC}$ & Normal & $\mathrm{PC}$ & Normal & \\
\hline- & 16 & 1 & 16 & 3 & 32 & 4 & $<0.01$ \\
\hline+ & 9 & 7 & 12 & 9 & 21 & 16 & \\
\hline++ & 1 & 18 & 1 & 17 & 2 & 35 & \\
\hline
\end{tabular}

Normal pancreatic tissue consists of surrounding non-neoplastic pancreatic tissue. LIFR, leukemia inhibitory factor receptor; PC, pancreatic cancer.

pre-coated with Matrigel (BD Biosciences, San Diego, CA, USA) according to the manufacturer's protocols before cell solution was added to the upper chamber. The lower chambers included $0.6 \mathrm{ml}$ of medium containing $10 \%$ FBS as a chemoattractant. After $24 \mathrm{~h}$ of culturing, the chambers were fixed with $10 \%$ methanol and stained with $0.5 \%$ crystal violet solution for $15 \mathrm{~min}$, and then washed by PBS. Cells in the lower chamber were observed and counted in six random fields under an inverted microscope.

Wound healing assay. Cells were layered onto 6-well plates and cultured to confluency. The 200- $\mu 1$ pipette tips were used to scratch three separate wounds on the monolayer of cells. Plates were washed with fresh medium after the cells had been cultured for 0,12 or $24 \mathrm{~h}$, and then photographed. The distances between wound edges were measured.

In vivo tumorigenesis and metastasis assays by micro-PET/CT. PC xenografts were established in nude mice. Four-week-old male BALB/c nude mice were purchased from the Institute of Zoology, Chinese Academy of Sciences of Shanghai. The animal research was approved by the Ethics Committee of Ruijin Hospital, Shanghai Jiao Tong University School of Medicine. All experiments were performed in accordance with the official recommendations of the China Zoological Society and animals received humane care according to the criteria outlined in the 'Guide for the Care and Use of Laboratory Animals'. Briefly, Capan-1/sh, Capan-1/nc, PATU-8988/vector and PATU-8988/LIFR cells were re-suspended in PBS ( $\mathrm{pH}$ 7.4). The suspension, containing $1 \times 10^{6}$ cells, was subcutaneously injected into the right flank of nude mouse. The length (L) and width (W) of each tumor were measured every 7 days with a digital caliper, and the tumor volume was calculated using the formula: Tumor volume $=\left(\right.$ Width $^{2} \mathrm{x}$ Length $) / 2$. Mice were sacrificed by cervical decapitation 5 weeks after injection. Tumors were weighed and fixed for hematoxylin and eosin (H\&E) and immunohistochemical staining. The tumorigenic experiments in vivo were performed with 5 mice in each group.

The tail vein injection assay was employed to evaluate the role of LIFR in tumor metastases in vivo. The stable clones of Capan-1/sh, Capan-1/nc, PATU-8988/vector and PATU-8988/LIFR were injected into athymic nude mice via tail veins. After 6 weeks, the lung metastasis lesions were detected by micro-PET/CT. PET/CT imaging was performed by the Department of Nuclear Medicine, Ruijin Hospital, Shanghai Jiao Tong University School of Medicine according to Meng's method (16). Then, the mice were sacrificed and all the suspicious lung metastasis sites were evaluated by histological examination.

Statistical analyses. Statistical analyses were performed using SPSS 13.0 software (SPSS, Inc., Chicago, IL, USA). For comparison among groups, an analysis of variance (ANOVA) and Student's t-test were used. Differences between tumor volumes were assessed by Mann-Whitney U test. The Chi-square test was used for the comparison of categorical data. A P-value $<0.05$ was considered to indicate a statistically significant result.

\section{Results}

LIFR expression is downregulated in PC tissues and is associated with clinicopathological parameters. To illuminate the expression of LIFR in multiple cancer tissues, Oncomine data-mining analysis analyses were first performed. Pei Pancreas Statistics confirmed that with all four probes testing it showed significantly decreased levels of LIFR mRNA in PC compared with that in normal pancreas tissues (Fig. 1A and B, available at Oncomine website).

To confirm our above findings, we then examined the expression level of LIFR in 26 PC clinical cases and 29 PC tissue microarrays, including 53 pancreatic duct adenocarcinomas and 2 pancreatic adenosquamous carcinomas. Immunohistochemical staining of 55 paired tissues showed that LIFR expression was significantly lower in the tumor tissues than that in the non-tumor tissues (negative, 32:4, positive, 21:16; strong positive, 2:35) (Table I) (Fig. 1C and D). Furthermore, LIFR was downregulated in $70.9 \%(39 / 55)$ of the PC cases. These results were consistent with those from the Oncomine analyses. PC tissue microarray was further employed to examine the correlation between LIFR expression and clinicopathological features. The results showed that downregulated LIFR was associated with lymph node metastasis $(\mathrm{P}=0.014)$, local invasion $(\mathrm{P}=0.047)$ and TNM stage $(\mathrm{P}=0.002)$, but not with other clinicopathological factors including gender, age and tumor location (Table II). Further analysis showed that only TNM stage of the patients was correlated to LIFR expression $(\mathrm{P}=0.002)$, not gender, age, tumor location or tumor size (Table III). All these findings 
Table II. Association between LIFR expression and clinicopathological factors of the PC patients.

\begin{tabular}{|c|c|c|c|c|}
\hline \multirow[b]{2}{*}{ Variables } & \multirow[b]{2}{*}{$\begin{array}{l}\text { No. of } \\
\text { cases }\end{array}$} & \multicolumn{2}{|c|}{ LIFR immunostaining } & \multirow[b]{2}{*}{ P-value } \\
\hline & & $\begin{array}{c}\text { Positive } \\
(\mathrm{n}=13)\end{array}$ & $\begin{array}{l}\text { Negative } \\
(\mathrm{n}=16)\end{array}$ & \\
\hline Gender & & & & 0.730 \\
\hline Male & 21 & 9 & 12 & \\
\hline Female & 8 & 4 & 4 & \\
\hline Age (years) & & & & 0.089 \\
\hline$>60$ & 15 & 9 & 6 & \\
\hline$\leq 60$ & 14 & 4 & 10 & \\
\hline Tumor location & & & & 0.244 \\
\hline Head & 19 & 10 & 9 & \\
\hline Tale & 10 & 3 & 7 & \\
\hline Tumor size $(\mathrm{cm})$ & & & & 0.051 \\
\hline$>45$ & 10 & 2 & 8 & \\
\hline$\leq 45$ & 19 & 11 & 8 & \\
\hline T stage & & & & 0.033 \\
\hline $\mathrm{T} 1$ & 6 & 5 & 1 & \\
\hline $\mathrm{T} 2+\mathrm{T} 3$ & 23 & 8 & 15 & \\
\hline $\begin{array}{l}\text { Lymph node } \\
\text { metastasis }\end{array}$ & & & & 0.014 \\
\hline Negative & 20 & 12 & 8 & \\
\hline Positive & 9 & 1 & 8 & \\
\hline Local invasion & & & & 0.047 \\
\hline Negative & 12 & 8 & 4 & \\
\hline Positive & 17 & 5 & 12 & \\
\hline Distant metastasis & & & & 0.119 \\
\hline Negative & 23 & 12 & 11 & \\
\hline Positive & 6 & 1 & 5 & \\
\hline TNM stage & & & & 0.002 \\
\hline IA-IB & 13 & 10 & 3 & \\
\hline IIA-IV & 16 & 3 & 13 & \\
\hline
\end{tabular}

Positive LIFR expression included all positive cases, such as weak and strong. LIFR, leukemia inhibitory factor receptor; PC, pancreatic cancer; TNM, tumor-node-metastasis.

suggested that downregulation of LIFR plays a critical role in PC development and LIFR may be an independent prognostic factor of PC.

LIFR negatively regulates the proliferation of PC cells. Based on the above results, to establish a paired differential expression model for further study, we first quantitatively analyzed the expression of LIFR in a series of PC cell lines (CFPAC-1, PATU-8988, Capan-1, SW-1990, BxPC-3 and PANC-1). Among these PC cells, the protein level of LIFR in Capan-1 cells was higher than that in the other PC cells and LIFR in highly aggressive PATU-8988 cells was the lowest (Fig. 2A). Therefore, Capan-1 cells were selected for the knockdown assay to reveal the role of LIFR in the following study. The
Table III. Association between TNM stage and clinicopathological factors of the PC patients.

\begin{tabular}{|c|c|c|c|c|}
\hline \multirow[b]{2}{*}{ Variables } & \multirow[b]{2}{*}{$\begin{array}{l}\text { No. of } \\
\text { cases }\end{array}$} & \multicolumn{2}{|c|}{ TNM stage } & \multirow[b]{2}{*}{ P-value } \\
\hline & & $\begin{array}{l}\text { IA-IB } \\
(n=13)\end{array}$ & $\begin{array}{l}\text { IIA-IV } \\
(n=16)\end{array}$ & \\
\hline \multicolumn{5}{|l|}{ Gender } \\
\hline Male & 21 & 10 & 11 & 0.624 \\
\hline Female & 8 & 3 & 5 & \\
\hline \multicolumn{5}{|c|}{ Age (years) } \\
\hline$>60$ & 15 & 8 & 7 & 0.340 \\
\hline$\leq 60$ & 14 & 5 & 9 & \\
\hline \multicolumn{5}{|c|}{ Tumor location } \\
\hline Head & 19 & 11 & 8 & 0.051 \\
\hline Tale & 10 & 2 & 8 & \\
\hline \multicolumn{5}{|c|}{ Tumor size (cm) } \\
\hline$>45$ & 10 & 2 & 8 & 0.051 \\
\hline$\leq 45$ & 19 & 11 & 8 & \\
\hline \multicolumn{5}{|c|}{ LIFR immunostaining } \\
\hline Positive & 13 & 10 & 3 & 0.002 \\
\hline Negative & 16 & 3 & 13 & \\
\hline
\end{tabular}

Positive LIFR expression included all positive cases, such as weak and strong. TNM, tumor-node-metastasis; LIFR, leukemia inhibitory factor receptor; $\mathrm{PC}$, pancreatic cancer.

lentiviral-mediated shRNA was employed to knockdown LIFR in the Capan-1 cells. Meanwhile, we used the pWPIGFP/LIFR vector to generate a PATU-8988/LIFR cell line ectopically overexpressing LIFR. After the establishment of stable clones of shRNA-mediated knockdown of LIFR in the Capan-1/sh cells and effective overexpression of LIFR in the PATU-8988/LIFR cells, western blotting was employed to confirm the expression level of LIFR in the Capan-1/sh and PATU-8988/LIFR cells (Fig. 2B and C). Colony formation assays indicated that silencing of endogenous LIFR in the Capan-1/sh cells significantly increased the ability of colony formation compared with the mock cells $(328.5 \pm 27.5$ vs. 106.5 \pm 8.5 ; $\mathrm{P}=0.016$ ) (Fig. $2 \mathrm{D}$ and $\mathrm{E}$ ). On the contrary, overexpression of LIFR in the PATU-8988/LIFR cells markedly impaired the ability of colony formation (177.5 \pm 9.5 vs. $438.0 \pm 15.0 ; \mathrm{P}=0.005$ ) (Fig. $2 \mathrm{~F}$ and $\mathrm{G}$ ). Collectively, these results indicated that LIFR may play a functional role in pancreatic carcinogenesis.

LIFR negatively regulates cell metastasis and invasion of $P C$ cells in vitro. A close correlation between clinical invasive characteristics in PC patients and expression level of LIFR was noted. This suggested that LIFR may play a negative regulatory role in $\mathrm{PC}$ tumor metastasis. To further confirm the effect of LIFR on the metastasis of PC cells, Transwell migration and invasion assays were performed. As shown in Fig. 3A and B, migration and invasion in the Capan-1/sh cells were significantly enhanced after silencing of endogenous LIFR (migration, $750.0 \pm 18.0$ vs. $382.5 \pm 15.5, \mathrm{P}=0.004$; 
A

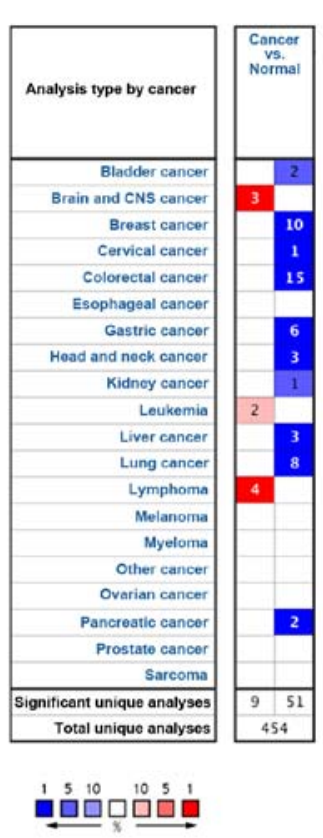

B

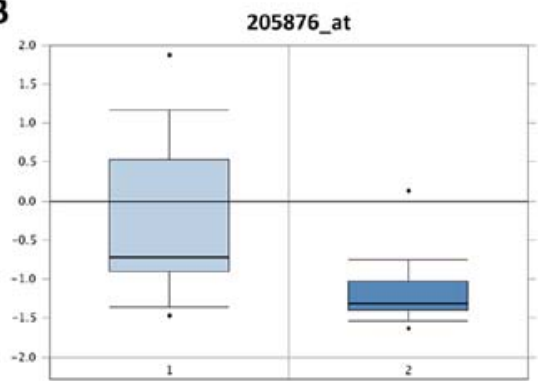

225575_at

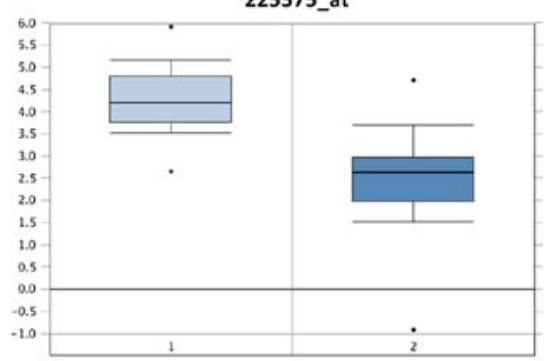

1. Pancreas $(n=16)$

2. Pancreatic carcinoma $(n=36)$
C

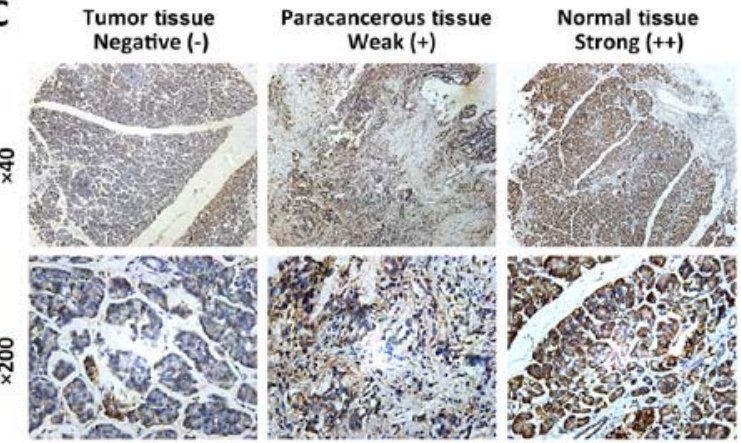

D

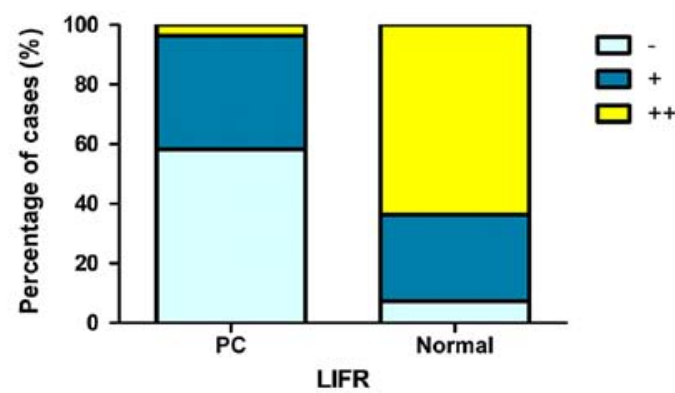

LIFR

Figure 1. LIFR expression is downregulated in PC tissues. (A) Downregulation of LIFR was found in 10 of 20 cancer types. (B) The level of LIFR mRNA was significantly decreased from pancreatic cell dysplasia to PC in all of the four probes (205876_at, 225571_at, 225575_at and 227771_at) in the Pei Pancreas Statistics. (C) Characterization of LIFR protein expression in human PC, paracancerous and paired adjacent non-tumor tissues by immunohistochemical staining. (D) Immunostaining score distribution of LIFR expression in PC $(n=55)$ and normal pancreatic tissues $(n=55)$.

A

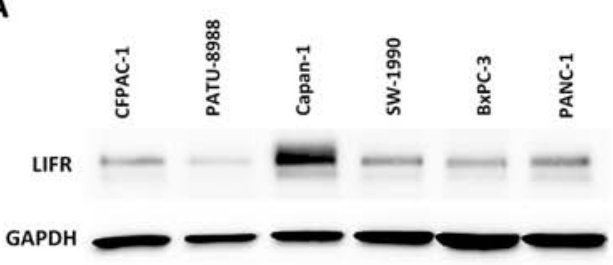

D

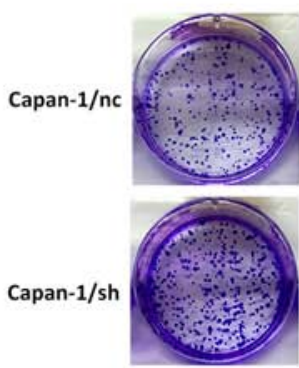

$\mathrm{E}$

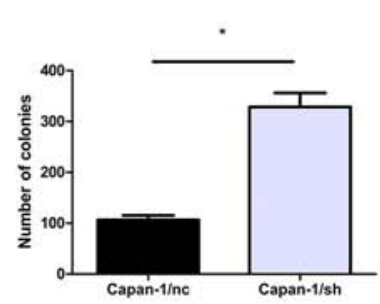

B

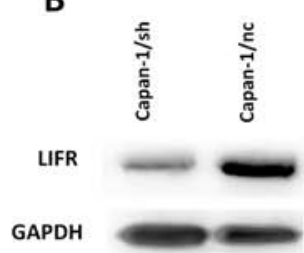

$\mathbf{F}$

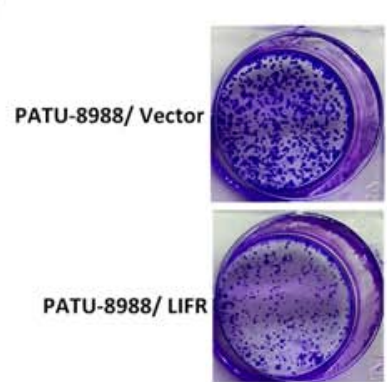

225571_at

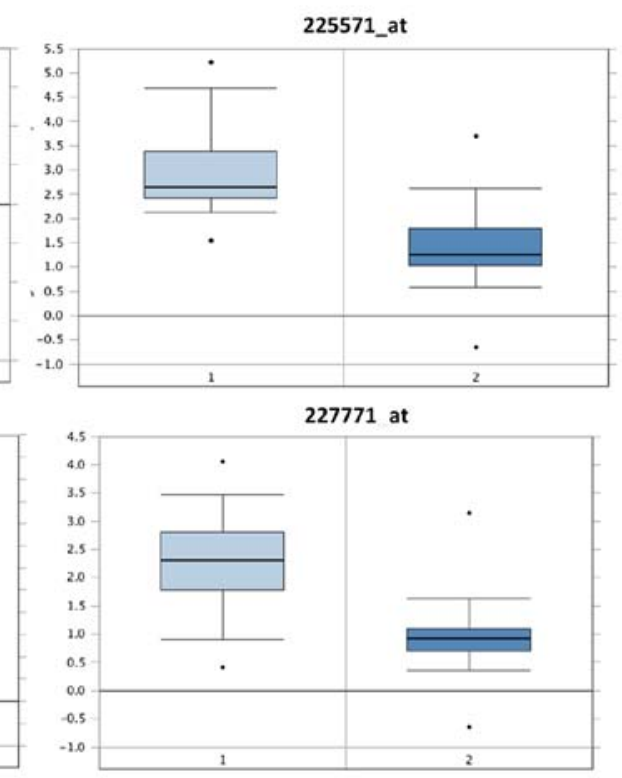

227771 at

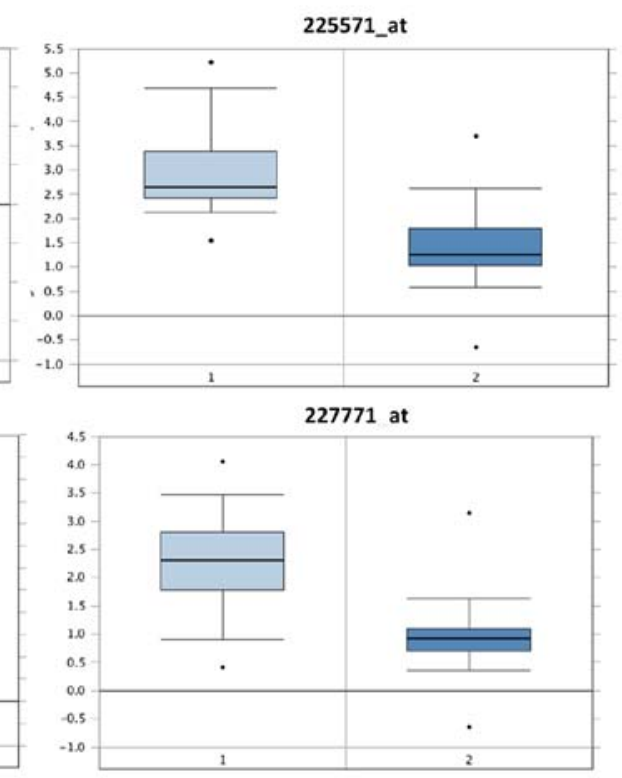


A

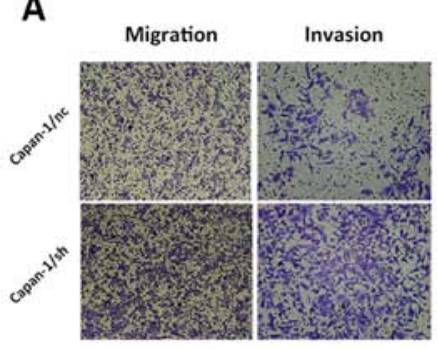

B

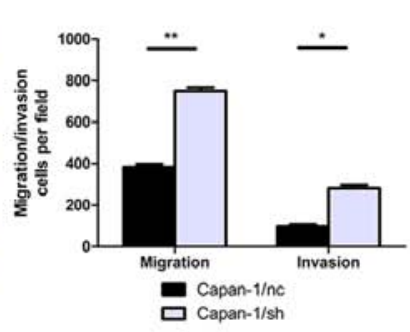

E

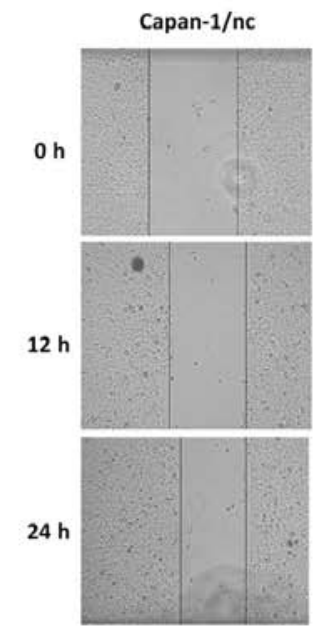

Capan-1/sh

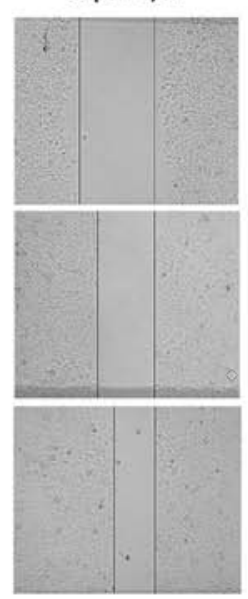

$\mathbf{F}$

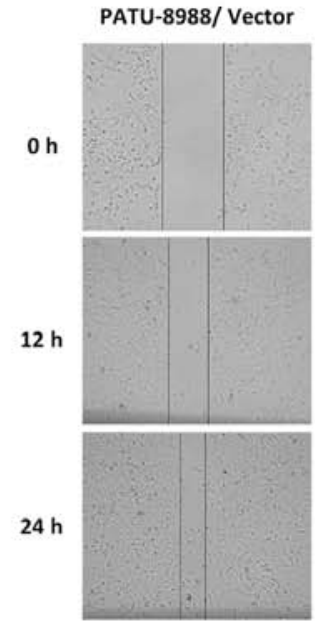

C

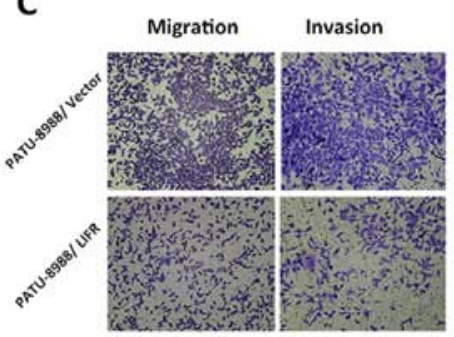

D

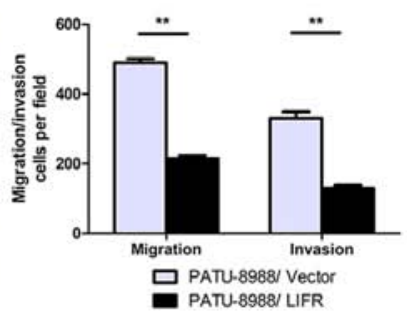

G

Figure 3. LIFR negatively regulates the metastasis of PC cells in vitro. (A and B) After infection of lentivirus-mediated LIFR shRNA, LIFR expression was detected by western blotting in the Capan-1 cells, respectively. Metastatic potentials were analyzed by Transwell assays. (C and D) After LIFR vector transfection, LIFR expression was detected by western blotting in the PATU-8988 cells, respectively. Metastatic potentials were analyzed by Transwell assays. (E and F) Wound healing assays with PC cells. Microscopic observations were recorded 0, 12 and $24 \mathrm{~h}$ after scratching the cell surface. A representative image from every independent experiment is shown. $(\mathrm{G})$ The distances between wound edges of the $\mathrm{PC}$ cells at 0,12 and $24 \mathrm{~h}$. ${ }^{*} \mathrm{P}<0.05$; ${ }^{* *} \mathrm{P}<0.01$.

invasion, $281.0 \pm 16.0$ vs. $97.0 \pm 10.0, \mathrm{P}=0.010)$. In contrast, by overexpressing LIFR in PC cells, the migratory and invasive abilities of the PATU-8988/LIFR cells were apparently reduced (migration, $215.0 \pm 9.0$ vs. $490.0 \pm 11.0, \mathrm{P}=0.003$; invasion, $130.5 \pm 7.5$ vs. $330.5 \pm 18.5, \mathrm{P}=0.010$ ) (Fig. $3 \mathrm{C}$ and $\mathrm{D}$ ).

Furthermore, we quantitatively investigated the effect of LIFR on migratory ability by wound healing assay. The results in Fig. 3E and G showed that silencing of LIFR significant shortened the distance between the wound edge in the Capan- $1 /$ sh cells compared with the Capan- $1 /$ nc cells $(\mathrm{P}=0.034)$. Consistently, we observed a longer distance in wound healing after overexpressing LIFR in the PATU-8988/LIFR cells $(\mathrm{P}=0.013)$ (Fig. 3F and $\mathrm{G})$. Taken together, these results indicated that the aggressive and highly metastatic phenotype of PC cells could be regulated by LIFR in vitro.

LIFR negatively regulates tumorigenesis and metastasis of PC cells in vivo. For in vivo confirmation, a recombinant lentivirus harboring LIFR was transfected into the PATU-8988 cells. The stable clone expressing ectopic PATU-8988/LIFR was subcutaneously injected into the flank of each athymic nude mouse, and an equal volume of cells transfected with the empty vector was injected into the opposite flank of the same mouse as the negative control. As shown in Fig. 4A and B, the PATU-8988/ LIFR cells caused smaller tumor masses than the mock vector control after 5 weeks of observation (PATU-8988/LIFR,
$47.9 \pm 12.5 \mathrm{~mm}^{3}$; PATU-8988/vector, $\left.407.2 \pm 143.2 \mathrm{~mm}^{3} ; \mathrm{P}<0.05\right)$. Meanwhile, tumors derived from the offspring subclones with LIFR-overexpressing cells were significantly lighter than those in the control group (PATU-8988/LIFR, 0.106 $\pm 0.032 \mathrm{~g}$; PATU-8988/vector, $0.828 \pm 0.273 \mathrm{~g} ; \mathrm{P}<0.05$ ) (Fig. 4C). Furthermore, tumor sections from the nude mouse model were immunohistochemically stained for Ki67. We observed that Ki67 expression was decreased in the PATU-8988/LIFR group compared with the PATU-8988/vector group (Fig. 4D). As expected, opposing results were observed in the LIFR-silenced group compared with the control group. Tumors derived from the offspring subclones with inhibited LIFR were significantly larger and heavier than those in the control after 5 weeks of observation (tumor volume, $355.5 \pm 26.6$ vs. $163.1 \pm 25.3 \mathrm{~mm}^{3}$, $\mathrm{P}<0.01$; tumor weight, $0.712 \pm 0.055$ vs. $0.326 \pm 0.052 \mathrm{~g}$, $\mathrm{P}<0.01$ ) (Fig. 4B and $\mathrm{C}$ ). Thus, the data from the in vitro and in vivo experiments revealed that LIFR negatively regulates the tumorigenesis of PC cells.

Based on the results above, an in vivo model was employed to further confirm the effect of LIFR on tumor metastasis. Stably transfected Capan-1/sh cells $\left(1 \times 10^{6}\right.$ cells) were injected into the nude mouse via tail vein to observe the distant metastasis of PC cells in vivo. After 6 weeks, we observed that the weight of mice in the Capan-1/sh group was significantly lower than that in the Capan-1/nc group (Fig. 4E; $\mathrm{P}<0.01$ ). Lung metastasis was then examined by micro/PET-CT and 
A

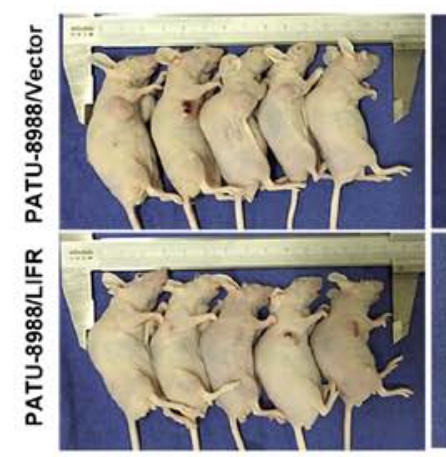

C

E

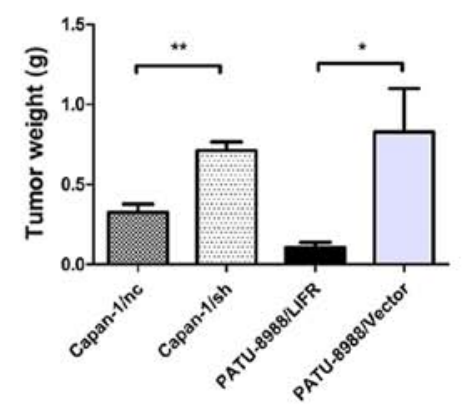

$\mathbf{F}$
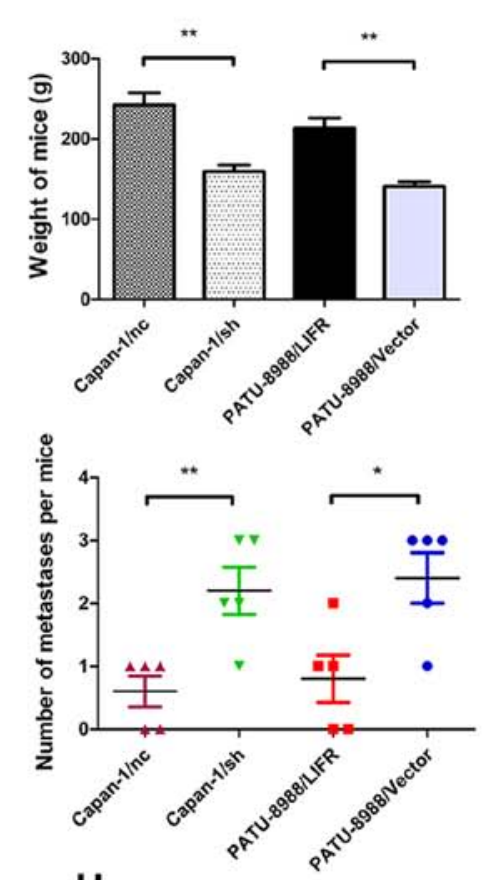

B

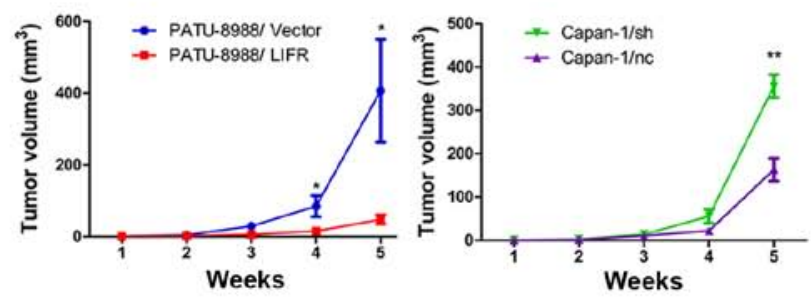

D

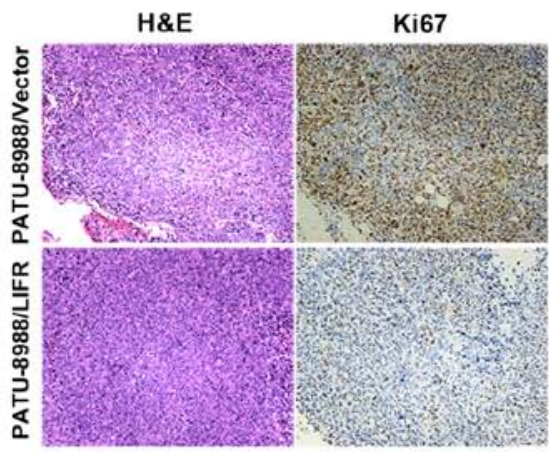

G
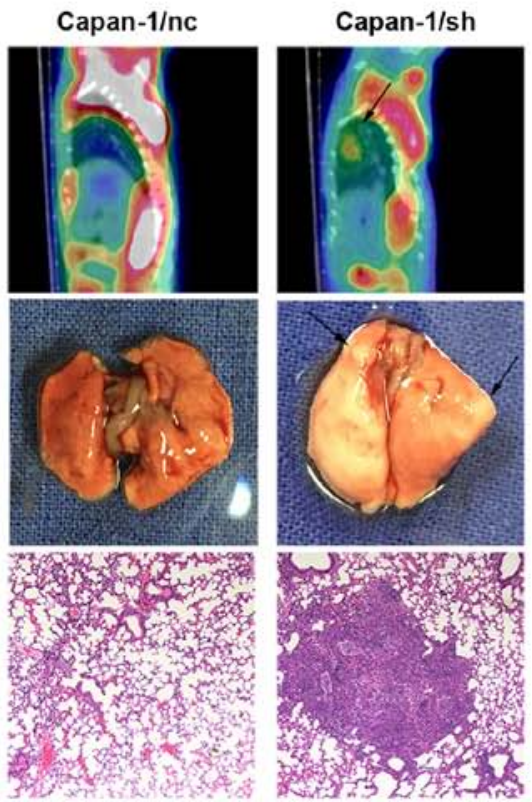

H
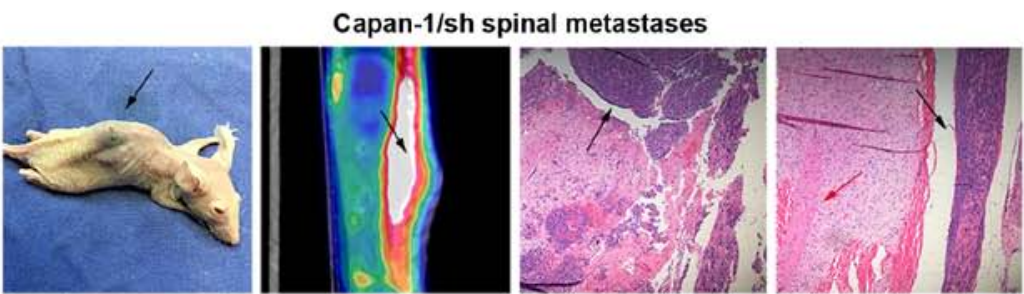

Figure 4. LIFR negatively regulates tumorigenesis and metastasis of PC cells in vivo. (A) Typical images of the xenograft tumors. (B) Xenograft tumor volumes were measured every 7 days. (C) Average weight of the xenograft tumors in nude mice. (D) Expression of Ki67 in the xenograft tumors by immunohistochemistry (magnification, x40). (E) Average weight of nude mice after being injected with PC cells into the tail veins. (F) The number of tumor nodules in each of the five nude mice after being injected with PC cells into the tail vein for 6 weeks. (G) Typical images of the effect on lung metastases of PC cells via tail vein injection. ${ }^{18} \mathrm{~F}$-FLT micro-PET/CT images of mice are shown at the top. The arrow indicates the ${ }^{18} \mathrm{~F}-\mathrm{FLT}$ uptake positivity in thoracic metastatic lesions. Representative images of lung metastases are shown in the middle panel and pathological study in the bottom panel. The arrows indicate metastatic tumors. (H) Spinal metastases in one mouse in the Capan-1/sh group. The black arrows indicate spinal metastases in the nude mouse by micro-PTC/CT and pathological study. The red arrow indicates nerve fibers in the pathological study. Tumor invasion of surrounding nerve fibers. ${ }^{*} \mathrm{P}<0.05$; ${ }^{* *} \mathrm{P}<0.01$. 
A

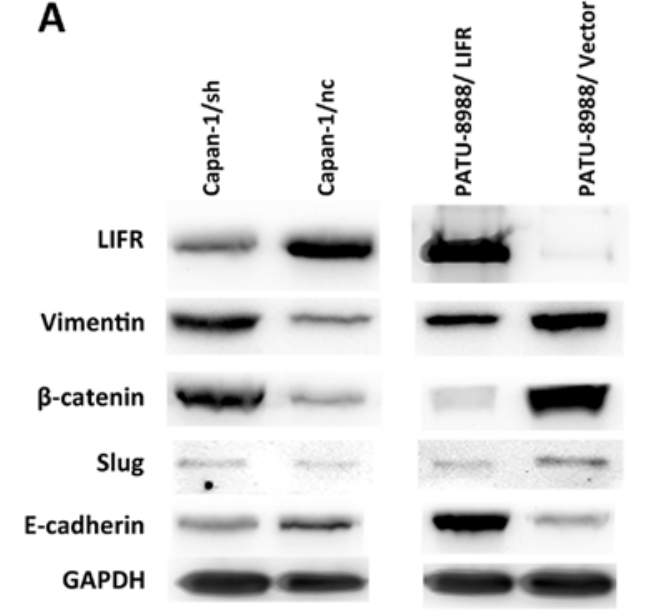

B

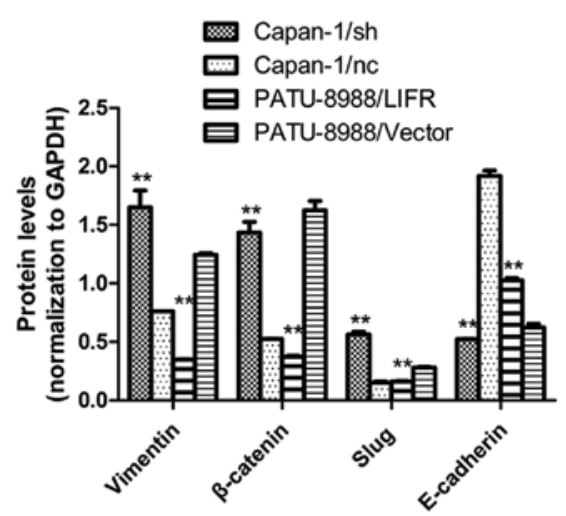

Figure 5. LIFR negatively regulates the metastasis of PC via epithelial to mesenchymal transition. (A and B) Western blot analyses showed that overexpression of LIFR in PATU-8988 cells could significantly decreased the expression of vimentin, $\beta$-catenin and slug and increased the expression of E-cadherin. Opposing results were observed in the LIFR-silenced groups. GAPDH was used as a loading control. ${ }^{*} \mathrm{P}<0.05 ;{ }^{* *} \mathrm{P}<0.01$.

confirmed by pathological study. Intriguingly, $5 / 5$ mice injected with Capan-1/sh cells developed larger and greater numbers of nodules of metastatic lung tumors whereas 3 metastatic tumors out of 5 were observed in the Capan-1/nc mice (Fig. 4F; $\mathrm{P}<0.01)$. The presence of lung metastases in these mice was also confirmed by histological analysis (Fig. 4G). Opposing results were observed in the LIFR-overexpressing groups compared with the control groups (Fig. 4E and F). In addition, we identified spinal metastases in one mouse from the Capan-1/sh group, and none in the Capan-1/nc group (Fig. 4H). Based on the in vitro and in vivo data, the results supported that LIFR negatively regulated the metastasis of PC cells both in vitro and in vivo.

LIFR negatively regulates cell metastasis and invasion of $P C$ via epithelial to mesenchymal transition. Research has demonstrated that epithelial-mesenchymal transition (EMT) is associated with cancer metastasis. Based on the results above, we further studied the possible molecular mechanisms by which LIFR contributes to PC cell proliferation and migration. As downstream regulation pathways of EMT, the activation of vimentin, $\beta$-catenin, slug and E-cadherin was detected to explore the underlying regulatory network. As shown in Fig. 5A and B, overexpression of LIFR in the PATU-8988/LIFR cells significantly inhibited the expression of vimentin, $\beta$-catenin and slug and induced the expression of E-cadherin. Opposing results were observed in the LIFR-silenced groups compared with the control groups (Fig. 5A and B). Collectively, these findings suggest that LIFR negatively regulates the metastasis of PC cells via EMT.

\section{Discussion}

Gearing et al originally isolated cDNA clones encoding leukemia inhibitory factor receptor (LIFR) by expression screening of a cDNA library using radioiodinated LIF as a probe (17). LIFR was structurally related to the IL-6 signal transducer and was found to belong to the gp130 receptor family (18). Therefore, LIFR plays broad roles in cell proliferation, cell differentiation and maintenance of stem cell pluripotency. LIFR was identified in several human malignancies and was found to be a metastasis suppressor in hepatocellular carcinoma and breast cancer $(11,14)$. Nandy et al reported that inhibition of LIFR induced by miR-125a activated the JAK2-STAT3 pathway stimulating a pro-carcinogenic molecular event in non-malignant breast epithelial stem cells along with inactivation of Hippo-TAZ signaling (19). In the present study, we firstly observed that the expression level of LIFR was significantly lower in the pancreatic cancer (PC) tumor tissues than that in the non-tumor tissues in 55 pairs of PC tissues. The results showed that LIFR expression was significantly lower in tumor tissues than that in non-tumor tissues. Furthermore, the downregulation of LIFR was associated with local invasion, lymph node metastasis and TNM stage, but not with the other clinicopathological factors. Although the primary results above indicated that LIFR may function in regulating the metastasis of PC, the detailed role and related mechanism require further elucidation.

Compared to other cancers, the metastases of PC, such as perineural invasion, bone metastasis, are more common and can be observed in up to $80 \%$ of cases (20-23). As a result, regional invasion and distant metastasis are the most common reasons causing the poor prognosis of PC $(24,25)$. As a possible metastasis suppressor in several cancers, LIFR plays crucial roles in cell proliferation and differentiation. To pinpoint the role of LIFR in PC, we initially investigated the influence of LIFR overexpression on colony formation ability in PC cells of both in vitro and in vivo. The results showed that overexpression of LIFR significantly inhibited the growth of PATU-8988/LIFR cells. In contrast, silencing of LIFR promoted propagation ability in Capan-1/sh PC cells. Our results were consistent with those found in hepatocellular carcinoma (13). Considering the importance of colony formation in tumorigenesis, our results suggested that LIFR may play an important role in the malignant transformation of PC. In contrast, wound healing was decreased significantly in the PATU-8988/LIFR cells and accelerated in the Capan-1/sh cells. Meanwhile, more Capan-1/sh cells infiltrated the Transwell gel and membrane 
to the lower chamber than the control groups. Consistently, only a small portion of PATU-8988/LIFR cells penetrated the membrane, compared with the control PATU-8988/vector cells. Based on the results in vitro, the nude mouse model was employed to confirm these results. The xenograft and lung metastasis models were established by subcutaneous and tail vein injection. Evidenced by digital caliper and micro-PET/ $\mathrm{CT}$, the tumor volume, weight and the lung metastatic nodules were increased by LIFR silencing and inhibited by overexpression. Thus, the results in vivo and in vitro clearly supported the notion that LIFR negatively regulates the tumorigenesis and metastasis of PC cells. Notably, in the present study, one out of five mice in the LIFR silenced group showed spinal metastases after eight-week injection via the tail vein, emphasizing the role of LIFR as a metastasis suppressor. Altogether, our findings revealed that LIFR could regulate the metastasis of PC cells and LIFR may serve as a potential marker for aggressive phenotype and as a therapeutic target.

Numerous studies have linked both migration and invasion to epithelial-mesenchymal (EMT)-like transition (26-28). Early steps of metastasis, particularly the early steps of hematogenous metastasis and lymphatic metastasis, have been linked to EMT (29). EMT markers are found at the invading front of several cancers, including colorectal, gastric, mammary and endometrial cancers (30-32). In the present study, we found that silencing of LIFR significantly increased the expression of vimentin, $\beta$-catenin and slug. Meanwhile, the expression of E-cadherin was significantly decreased. Opposing results were observed in the LIFR-overexpressing groups compared with the control groups. Slug triggers the steps of partial separation at cell-cell borders, cell spreading and desmosomal disruption, which are initial and essential parts of the EMT process (33), while $\beta$-catenin is a key component in the E-cadherin cell adhesion complex and the microtubule network (34). The morphological transition of cancer cells from an epithelial to a fibroblastic appearance is accompanied by scattering and directional migration toward serum factors, a loss of epithelial markers, such as E-cadherin, a gain of mesenchymal cell markers, such as vimentin, which essentially lead to metastasis. Together the above findings suggest that LIFR negatively regulates the metastasis of PC via EMT-like transition.

Furthermore, Shah et al (35) and Wang et al (36) found that the Notch signaling pathway was linked with the EMT phenotype of gemcitabine-resistant PC cells. This suggests that LIFR plays an important role in the chemoresistance of PC and could be a potential therapeutic approach for the treatment of metastatic chemoresistant PC. Our ongoing study will investigate the exact mechanism of LIFR as a tumor suppressor.

In conclusion, LIFR functions importantly in the tumorigenesis and metastasis of PC and the EMT regulation pathway may underlie the mechanism. Our research thereby provides new insight into PC metastasis and the function of LIFR.

\section{Acknowledgements}

The present study was supported by the Natural Science Foundation of China (81172326), and the Shanghai Charity Foundation for Cancer Research.

\section{References}

1. Torre LA, Bray F, Siegel RL, Ferlay J, Lortet-Tieulent J and Jemal A: Global cancer statistics, 2012. CA Cancer J Clin 65: 87-108, 2015

2. Jemal A, Bray F, Center MM, Ferlay J, Ward E and Forman D: Global cancer statistics. CA Cancer J Clin 61: 69-90, 2011.

3. Ryan DP, Hong TS and Bardeesy N: Pancreatic adenocarcinoma. N Engl J Med 371: 1039-1049, 2014.

4. Quaresma M, Coleman MP and Rachet B: 40-year trends in an index of survival for all cancers combined and survival adjusted for age and sex for each cancer in England and Wales, 1971-2011: A population-based study. Lancet 385: 1206-1218, 2015.

5. Konstantinidis IT, Warshaw AL, Allen JN, Blaszkowsky LS Castillo CF, Deshpande V, Hong TS, Kwak EL, Lauwers GY, Ryan DP, et al: Pancreatic ductal adenocarcinoma: Is there a survival difference for R1 resections versus locally advanced unresectable tumors? What is a 'true' R0 resection? Ann Surg 257: 731-736, 2013

6. Iacobuzio-Donahue CA, Fu B, Yachida S, Luo M, Abe H, Henderson CM, Vilardell F, Wang Z, Keller JW, Banerjee P, et al: DPC4 gene status of the primary carcinoma correlates with patterns of failure in patients with pancreatic cancer. J Clin Oncol 27: 1806-1813, 2009.

7. Kishimoto T, Akira S, Narazaki M and Taga T: Interleukin-6 family of cytokines and gp130. Blood 86: 1243-1254, 1995.

8. Alisoltani A, Fallahi H, Ebrahimi M, Ebrahimi M and Ebrahimie E: Prediction of potential cancer-risk regions based on transcriptome data: Towards a comprehensive view. PLoS One 9: e96320, 2014.

9. Salm F, Dimitrova V, von Bueren AO, Ćwiek P, Rehrauer H, Djonov V, Anderle P and Arcaro A: The phosphoinositide 3-kinase $\mathrm{p} 110 \alpha$ isoform regulates leukemia inhibitory factor receptor expression via c-Myc and miR-125b to promote cell proliferation in medulloblastoma. PLoS One 10: e0123958, 2015.

10. Liu SC, Tsang NM, Chiang WC, Chang KP, Hsueh C, Liang Y, Juang JL, Chow KP and Chang YS: Leukemia inhibitory factor promotes nasopharyngeal carcinoma progression and radioresistance. J Clin Invest 123: 5269-5283, 2013.

11. Chen D, Sun Y, Wei Y, Zhang P, Rezaeian AH, TeruyaFeldstein J, Gupta S, Liang H, Lin HK, Hung MC, et al: LIFR is a breast cancer metastasis suppressor upstream of the Hippo-YAP pathway and a prognostic marker. Nat Med 18: 1511-1517, 2012.

12. Tan X and Chen M: MYLK and MYL9 expression in non-small cell lung cancer identified by bioinformatics analysis of public expression data. Tumour Biol 12: 12189-12200, 2014.

13. Luo Q, Zhang Y, Wang N, Jin G, Jin H, Gu D, Tao X, Huo X, Ge T, Cong W, et al: Leukemia inhibitory factor receptor is a novel immunomarker in distinction of well-differentiated HCC from dysplastic nodules. Oncotarget 6: 6989-6999, 2015.

14. Luo Q, Wang C, Jin G, Gu D, Wang N, Song J, Jin H, Hu F, Zhang Y, Ge T, et al: LIFR functions as a metastasis suppressor in hepatocellular carcinoma by negatively regulating phosphoinositide 3-kinase/AKT pathway. Carcinogenesis 36: 1201-1212, 2015.

15. Seewoo V, Yang W, Du H, Wang J, Lin A, Shen B, Peng C, Li H and Qiu W: The different induction mechanisms of growth arrest DNA damage inducible gene $45 \beta$ in human hepatoma cell lines. Chemotherapy 58: 165-174, 2012.

16. Hong X, Bu L, Wang Y, Xu J, Wu J, Huang Y, Liu J, Suo H, Yang L, Shi Y, et al: Increases in the risk of cognitive impairment and alterations of cerebral $\beta$-amyloid metabolism in mouse model of heart failure. PLoS One 8: e63829, 2013.

17. Gearing DP, Thut CJ, VandeBos T, Gimpel SD, Delaney PB, King J, Price V, Cosman D and Beckmann MP: Leukemia inhibitory factor receptor is structurally related to the IL-6 signal transducer, gp130. EMBO J 10: 2839-2848, 1991.

18. Taga T, Narazaki M, Yasukawa K, Saito T, Miki D, Hamaguchi M, Davis S, Shoyab M, Yancopoulos GD and Kishimoto T: Functional inhibition of hematopoietic and neurotrophic cytokines by blocking the interleukin 6 signal transducer gp130. Proc Natl Acad Sci USA 89: 10998-11001, 1992.

19. Nandy SB, Arumugam A, Subramani R, Pedroza D, Hernandez K, Saltzstein E and Lakshmanaswamy R: MicroRNA-125a influences breast cancer stem cells by targeting leukemia inhibitory factor receptor which regulates the Hippo signaling pathway. Oncotarget 6: 17366-17378, 2015.

20. Li J, Ma Q, Liu H, Guo K, Li F, Li W, Han L, Wang F and Wu E: Relationship between neural alteration and perineural invasion in pancreatic cancer patients with hyperglycemia. PLoS One 6: e17385, 2011. 
21. Nakao A, Harada A, Nonami T, Kaneko T and Takagi H: Clinical significance of carcinoma invasion of the extrapancreatic nerve plexus in pancreatic cancer. Pancreas 12: 357-361, 1996.

22. Dai H, Li R, Wheeler T, Ozen M, Ittmann M, Anderson M, Wang Y, Rowley D, Younes M and Ayala GE: Enhanced survival in perineural invasion of pancreatic cancer: An in vitro approach. Hum Pathol 38: 299-307, 2007.

23. Keleg S, Büchler P, Ludwig R, Büchler MW and Friess $\mathrm{H}$ : Invasion and metastasis in pancreatic cancer. Mol Cancer 2: 14 , 2003.

24. Shimada K, Nara S, Esaki M, Sakamoto Y, Kosuge T and Hiraoka N: Intrapancreatic nerve invasion as a predictor for recurrence after pancreaticoduodenectomy in patients with invasive ductal carcinoma of the pancreas. Pancreas 40: 464-468, 2011.

25. Takahashi H, Ohigashi H, Ishikawa O, Gotoh K, Yamada T, Nagata S, Tomita Y, Eguchi H, Doki Y and Yano M: Perineural invasion and lymph node involvement as indicators of surgical outcome and pattern of recurrence in the setting of preoperative gemcitabine-based chemoradiation therapy for resectable pancreatic cancer. Ann Surg 255: 95-102, 2012.

26. Pasquier J, Abu-Kaoud N, Al Thani H and Rafii A: Epithelial to mesenchymal transition in a clinical perspective. J Oncol 2015: 792182, 2015.

27. Liu X, Yun F, Shi L, Li ZH, Luo NR and Jia YF: Roles of signaling pathways in the epithelial-mesenchymal transition in cancer. Asian Pac J Cancer Prev 16: 6201-6206, 2015.

28. Cohen EN, Gao H, Anfossi S, Mego M, Reddy NG, Debeb B, Giordano A, Tin S, Wu Q, Garza RJ, et al: Inflammation mediated metastasis: Immune induced epithelial-to-mesenchymal transition in inflammatory breast cancer cells. PLoS One 10: e0132710, 2015.

29. Lee JM, Dedhar S, Kalluri R and Thompson EW: The epithelialmesenchymal transition: New insights in signaling, development, and disease. J Cell Biol 172: 973-981, 2006.
30. Domínguez D, Montserrat-Sentís B, Virgós-Soler A Guaita S, Grueso J, Porta M, Puig I, Baulida J, Francí C and García de Herreros A: Phosphorylation regulates the subcellular location and activity of the snail transcriptional repressor. Mol Cell Biol 23: 5078-5089, 2003.

31. Rosivatz E, Becker KF, Kremmer E, Schott C, Blechschmidt K, Höfler $\mathrm{H}$ and Sarbia M: Expression and nuclear localization of Snail, an E-cadherin repressor, in adenocarcinomas of the upper gastrointestinal tract. Virchows Arch 448: 277-287, 2006

32. Brabletz T, Jung A, Reu S, Porzner M, Hlubek F, KunzSchughart LA, Knuechel R and Kirchner T: Variable beta-catenin expression in colorectal cancers indicates tumor progression driven by the tumor environment. Proc Natl Acad Sci USA 98: 10356-10361, 2001.

33. Savagner P, Yamada KM and Thiery JP: The zinc-finger protein slug causes desmosome dissociation, an initial and necessary step for growth factor-induced epithelial-mesenchymal transition. J Cell Biol 137: 1403-1419, 1997.

34. MacDonald BT, Tamai K and He X: Wnt/beta-catenin signaling: Components, mechanisms, and diseases. Dev Cell 17: 9-26, 2009.

35. Shah AN, Summy JM, Zhang J, Park SI, Parikh NU and Gallick GE: Development and characterization of gemcitabineresistant pancreatic tumor cells. Ann Surg Oncol 14: 3629-3637, 2007.

36. Wang Z, Li Y, Kong D, Banerjee S, Ahmad A, Azmi AS, Ali S, Abbruzzese JL, Gallick GE and Sarkar FH: Acquisition of epithelial-mesenchymal transition phenotype of gemcitabineresistant pancreatic cancer cells is linked with activation of the notch signaling pathway. Cancer Res 69: 2400-2407, 2009. 